\title{
Narrative review: preoperative localization techniques for small lung nodules
}

\author{
Stephanie H. Chang ${ }^{1}$, Travis C. Geraci ${ }^{1}$, Karleigh R. Johnson ${ }^{2}$, Amie J. Kent ${ }^{1}$, Robert J. Cerfolio ${ }^{1}$ \\ ${ }^{1}$ Division of Thoracic Surgery, Department of Cardiothoracic Surgery, New York University Langone Health, New York, NY, USA; ${ }^{2}$ School of \\ Medicine, University of Alabama-Birmingham, Birmingham, AL, USA \\ Contributions: (I) Conception and design: SH Chang, AJ Kent, RJ Cerfolio; (II) Administrative support: All authors; (III) Provision of study materials \\ or patients: All authors; (IV) Collection and assembly of data: All authors; (V) Data analysis and interpretation: All authors; (VI) Manuscript writing: \\ All authors; (VII) Final approval of manuscript: All authors. \\ Correspondence to: Stephanie H. Chang, MD. Division of Thoracic Surgery, Department of Cardiothoracic Surgery, New York University Langone \\ Health, 530 First Avenue, Suite 9V, New York, NY 10016, USA. Email: stephanie.chang@nyulangone.org.
}

\begin{abstract}
Minimally invasive surgery, both video-assisted and robotic-assisted, are increasingly used as the approach for surgical biopsy of small indeterminate pulmonary nodules. As the use and resolution of computed tomography (CT) scans increases, the need for surgical biopsy is rising concurrently. The combination of small nodules and minimally invasive surgical approaches can lead to difficulty in localizing these nodules intraoperatively. This results in a negative biopsy or conversion to open surgery for manual localization. In order to mitigate these issues, thoracic surgeons should be familiar with the available techniques for preoperative or peri-operative localization, as well as their respective advantages and disadvantages. Pre-operative localization options often require working closely with interventional radiology; specifically they can help with the placement of metallic markers or injection of tracers and dyes to assist with palpation or visualization of otherwise hard to locate nodules. Newer, non-radiologic, options for perioperative localization are also available. These include navigational bronchoscopy with indocyanine green (ICG) injection which is then visualized by a special intraoperative camera or image guided video-assisted thoracic surgery (iVATS) with placement of a marker in the same room and position used for resection. This paper will describe the most common techniques, their success rates and common complications, as well as the equipment needed for each approach. The decision regarding which described technique is ideal should be based on surgeon preference and familiarity with the procedure, as well as the available interventional radiologists and equipment at the institution.
\end{abstract}

Keywords: Pulmonary nodules; nodule localization; indocyanine green (ICG); preoperative localization; videoassisted thoracoscopic surgery (VATS); robotic-assisted thoracoscopic surgery (RATS)

Received: 26 April 2020; Accepted: 08 January 2021; Published: 25 November 2022.

doi: $10.21037 /$ ccts-20-90

View this article at: http://dx.doi.org/10.21037/ccts-20-90

\section{Introduction}

Due to the ever-increasing number of computed tomography (CT) scans, the rate of small pulmonary nodules is continually rising. The causes for increased CT scans are multi-fold. The National Lung Screening Trial demonstrated that use of low-dose CTs for high-risk patients showed a relative reduction in mortality of $20 \%$ from lung cancer (1), leading to the implementation of lung cancer screening programs. Chest CTs are also used for clinical staging in other malignancies (2), for preoperative evaluation prior to transcatheter aortic valve replacement (3), and for determination of coronary calcification (4). While most of these small nodules can be safely monitored, many other indeterminate nodules will need to undergo surgical biopsy to diagnosis to rule out malignancy. 
Concurrent to the increasing number of small lung nodules, thoracic surgery has progressed to more minimally invasive techniques. Video-assisted thoracoscopic surgery (VATS) and robotic-assisted thoracoscopic surgery (RATS) are increasingly being used for biopsy of small nodules and, if malignant, anatomic resection of early stage lung cancer (3). However, both VATS and RATS techniques can have trouble identifying small, nonsolid, or deep lung nodules as finger palpation is not feasible (4). In 1999, 54\% of VATS cases for lung nodule resection required conversion to open thoracotomy, with the majority of conversions done to help identify the lesion (5). In order to aid with intraoperative identification of these nodules for accurate surgical resection, different preoperative localization techniques are available. Knowledge of these techniques and their potential complications is essential for thoracic surgeons. This article will describe the main types of preoperative marker placement, preoperative tracer or dye injection, and intraoperative localization techniques. We present the following article in accordance with the Narrative Review reporting checklist (available at https://ccts.amegroups.com/ article/view/10.21037/ccts-20-90/rc).

\section{Methods}

All reviews, case series and reports, and trials involving localization of pulmonary nodules within the last 25 years that were listed on PubMed were reviewed and relevant articles were included in this work.

\section{Preoperative placement of markers}

Placement of metallic markers preoperatively has been shown to be very effective for localization of small pulmonary nodules. The three main options are microcoil, hookwire, and fiducial marker placement. Each of these techniques requires coordination with colleagues in interventional radiology to place these markers using CT guidance prior to the procedure.

\section{Microcoil placement}

The microcoil technique involves placement of a coil next to the desired lung nodule, with one end deep to the nodule and one end superficial to the nodule. Thus, the microcoil is contained within the lung parenchyma and can be located intraoperatively with fluoroscopy (6). Multiple studies have shown a high rate of successful identification of the target pulmonary nodule with subsequent adequate resection and diagnosis. In two different series the identification success rate was $100 \%$, Donahoe et al. in 63 patients and Rodrigues et al. in 124 patients $(7,8)$. When compared to patients not receiving preoperative localization, Finley et al. demonstrated percutaneous CT-guided microcoil localization prior to VATS led to complete resection of the nodule for diagnosis in $93 \%(27 / 29)$ of patients $v s$. $48 \%(13 / 27)$ in the control group (9). This study also demonstrated that microcoil preoperative localization was associated with a shorter operative time, decreased stapler use, and shorter mean hospital length of stay (9). Immediate confirmation of complete resection of the nodule can be determined with a plain radiographic image showing the microcoil in the specimen.

Though microcoil placement is a reliable marker, there are some complications associated with this technique. The previously mentioned studies described a $2 \%$ risk of preoperative pneumothorax (8), $1 \%$ risk of intraoperative episode of hemothorax (7), 1.6\% risk of hemoptysis (7), and $4 \%$ rate of microcoil dislodgement (8). In the patients with a displaced microcoil, an additional microcoil was required at the time of placement (8) which allowed for the $100 \%$ resection rate. While the rate of microcoil dislodgement was previously higher (10), the change in technique, with the coil being fully embedded within the lung parenchyma and not extending to the pleura, has led to more accurate placement (6).

\section{Hookwire placement}

Hookwire localization involves the percutaneous placement of a hook using CT guidance adjacent to, or deep to, the lesion, with the attached wire extending externally and taped to the skin. Intraoperative localization occurs using the wire to visualize the track to the nodule. Of note, the wire must be left with some slack at the skin, since a pneumothorax can cause the lung to pull away and dislodge the hook (6). The data for hookwire use also demonstrates high efficacy, with some studies showing $100 \%$ localization using this technique (11). Ciriaco et al. demonstrated a $92 \%(49 / 53)$ rate of identification of small nodules, though only $58 \%(31 / 53)$ of the patients had nodules that were not visible or palpable on the pleural surface (12). Similar to microcoils, the use of hookwires has been associated with decreased operative time (12). 
The hookwire method of nodule localization has similar complications to the microcoil, though at a higher rate. Pneumothorax rates after the localization procedure have been noted to be as high as $49 \%$ (206/417 of patients) directly after the procedure and up to $68 \%$ (284/417 of patients) 30 minutes after the procedure (13). Aspiration of the pneumothorax was required in $4.6 \%(19 / 417)$ of patients due to $>50 \%$ collapse or presence of symptoms (13). Other groups demonstrate a smaller rate of pneumothorax, ranging from $7.5 \%$ to $35.8 \%$, none of whom required a chest tube $(11,12)$. Wire displacement is potentially higher than microcoils, as it has been seen in all analyzed series, varying from $2.7 \%$ to $13 \%(11,14,15)$. For many of these displaced hookwires, the hematoma on the pleura of the surface was sufficient to guide the surgeon to successful resection of the specimen (12). Dislodgment often occurred during transport to the operating room or while positioning and prepping the patient on the operating table for surgery. Positioning the patient appropriately on the operating table and leaving a longer length of hookwire outside of the chest in order to allow the single ventilated lung to deflate without issue showed a reduced risk of dislodgment (12). Hemoptysis and pulmonary hematoma complications were also higher with one study showing 11/417 and 37/417 patients experiencing these respectively (13). Notably, hookwires are also associated with the risk of systemic air embolism. While one study demonstrated a $0.24 \%$ risk with spontaneous resolution (13), a case report described this complication with cardiovascular collapse and asystole directly after placement of the hookwire (16). CT images demonstrated a large quantity of gas in the ascending aorta, requiring Trendelenburg positioning and immediate resuscitation to prevent a mortality (16).

\section{Fiducial markers}

Similar to microcoils, radiopaque fiducial markers can be placed preoperatively in or adjacent to the target, with intraoperative identification using fluoroscopy. The gold markers are inert and rarely migrate if placed within the pulmonary parenchyma (10). This allows them to be placed on a different day than the procedure. Sancheti et al. demonstrated a $98 \%(57 / 58)$ success rate of nodule removal with the use of fiducials, with the mean size of $9.9 \pm 4.6 \mathrm{~mm}$ and mean depth from the visceral pleura of $18.7 \pm 12 \mathrm{~mm}$ (17). A smaller series demonstrated high efficacy as well, with a 95\% (19/20) success rate; the one failure being due to deployment of the marker within the pleural space (18). Fluoroscopy time was measured and was found to be a mean of 46 seconds with an associated mean radiation dose of $12.97 \mathrm{mGy}$ (18). A radiographic image of the removed lung is used to identify the presence of the fiducial in the specimen.

As with the other marker placements, there is a small rate of complications. A small series showed a $10 \%(2 / 20)$ rate of pneumothorax (18) vs. another which had no reported occurrences (17). No cases of hemoptysis were reported in the examined series, and only one case had the presence of a pleural hematoma (18). While the risk of fiducial migration is low, there was a $1.7 \%$ chance of migration in one series (17). More unique to fiducial markers is the risk of vascular embolization. Sancheti et al. demonstrated a $1.7 \%(1 / 58)$ chance of fiducial vascular embolization (17), with a case report from a different institution describing the fiducial embolizing through the pulmonary vein to the left ventricle (18). Though this patient had no long-term morbidity (19), there remains the potential risk of stroke with vascular embolization.

\section{Preoperative tagging with liquids}

Localization of small pulmonary nodules can be performed using CT-guided marking with liquids, such as methylene blue, barium, India ink, and radiotracers (10). Of these multiple options, the two most commonly used options are methylene blue injection and radiotracer injection. While the marker placements can occur within a various amount of time prior to surgical resection, tagging with methylene blue or radiotracers both require surgical resection within hours as the liquids diffuse over time which results in decreased localization accuracy (10).

\section{Methylene blue localization}

Methylene blue is a blue dye that has been used since the 1990s for tagging small pulmonary nodules. Using CT guidance, the blue dye is injected superficial to the nodule and along the tract to the pleural surface with the majority of the dye injected into the subpleural parenchyma, allowing for identification of the target area (Figure 1). Importantly, the methylene blue dye must not be spilled into the pleural cavity, as the area marked for resection may then be larger than necessary. Of the available localization techniques, it requires the least amount of equipment and remains the most cost effective.

In a study performed during 1992-1996, Vandoni et al. 


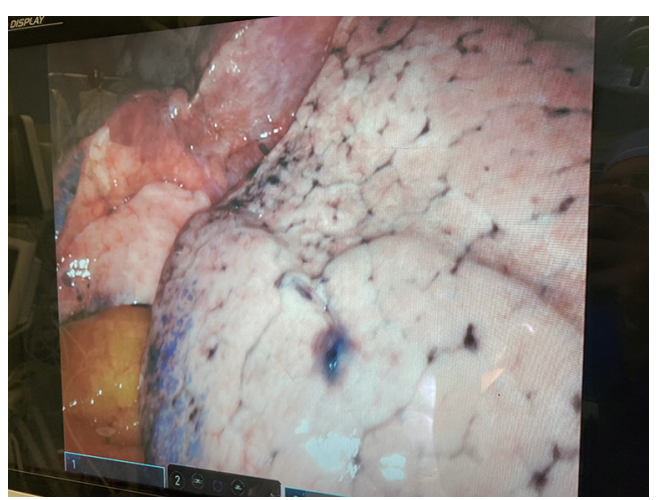

Figure 1 Intraoperative view during VATS with preoperative methylene blue localization. VATS, video-assisted thoracoscopic surgery.

used methylene blue for pulmonary nodules less than $2.5 \mathrm{~cm}$ that were not located on the pleural surface, with a $93 \%$ (50/54) success rate (20). Of these nodules, 91\% (49/54) were wedged out via VATS. They did note a $13 \%$ rate of post-injection pneumothorax prior to surgery, but these were clinically irrelevant and no intervention was needed. A more recent study evaluating 80 patients with lesions less than $2 \mathrm{~cm}$ demonstrated a $94 \%$ rate of localization with CT virtual simulation the day prior to surgery and methylene blue injection just prior to surgery (21). The average lesion size was $10.4 \pm 3.5 \mathrm{~mm}$ and average depth was $9.4 \pm 4.9 \mathrm{~mm}$.

While most studies did not report any complications there is a small risk of allergic reaction, $<1 \%(10)$. When evaluating different blue dyes that are used for sentinel lymph node mapping for other malignancies, methylene blue has the lowest rate of allergic reaction, which includes rashes, urticaria, or anaphylaxis (22). There have been no reported cases of anaphylaxis during preoperative localization for pulmonary nodules using methylene blue.

\section{Technetium 99 localization}

Several techniques using radiotracers have been used to localize small pulmonary lesions. Of these, technetium-99m (Tc-99m), the same radiotracer used in lung perfusion scans, has evolved as a safe and feasible option with reproducible, successful accuracy. While the technique requires coordination with radiology for preoperative injection, the radiotracer has a relatively long half-life, is stable in tissue, is easily localized with a gamma probe, and does not risk dislodgement.
In a two-stage, translational study from 2004, Daniel et al. reported the feasibility of using CT-guided transthoracic peritumoral injection of Tc-99m to localize small pulmonary lesions for subsequent resection (23). After radiographic injection of $\mathrm{Tc}-99 \mathrm{~m}$, intraoperative gamma probe localization was $100 \%$ successful (13 patients) in identifying the radiotracer during thoracoscopic surgery. In a large cohort of 211 patients undergoing thoracoscopic resection of sub-centimeter pulmonary nodules, Ambrogi et al. reported a localization accuracy of $99 \%$ (208/211) with Tc$99 \mathrm{~m}$ injection (24). All patients underwent surgical resection within 4 hours of CT-guided injection of radiotracer.

Regarding Tc-99m injection, there are limited complications. Daniel et al. had no reported complications from radiologic injection or surgical resection (23). In the series by Ambrogi et al., a small number of patients (10\%) developed a pneumothorax prior to resection, which did not affect surgical resection or postoperative recovery (24). Similar to methylene blue, wide radiotracer parenchymal diffusion or spillage into the pleural space prevented localization in a small number of patients (24). The authors note that radiotracer diffusion was more common in patients with severe emphysema, suggesting a relative contraindication to the technique.

\section{Concurrent operative localization}

More recent developments have led to different techniques that allow for small pulmonary nodule localization in the operating room, resulting in the use of a single anesthetic and procedure for marking and resection. The two most promising developments are injection of dyes using navigational bronchoscopy and placement of markers at the time of the operation.

\section{Near-infrared fluorescence (NIF) with indocyanine green (ICG)}

Methods of pulmonary nodule localization using electromagnetic navigational bronchoscopy allow surgeons to complete localization in the operating room. Utilizing NIF technology via thoracoscopic cameras, peritumoral injection of ICG contrast has emerged as an accurate and efficient method for localizing pulmonary nodules and, an additional benefit when it is administered intravenously (IV), ICG can also delineate the intersegmental plane.

Performing localization starts with a preoperative review 


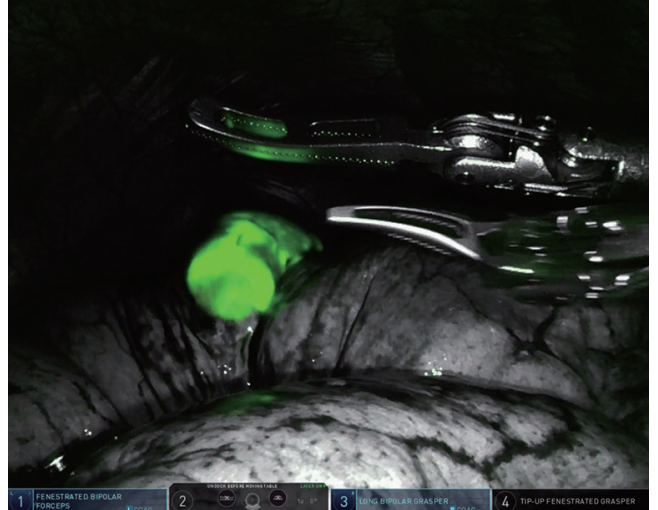

Figure 2 Intraoperative view during RATS after intraoperative navigational bronchoscopy with ICG contrast injection near the lesion. RATS, robotic-assisted thoracoscopic surgery; ICG, indocyanine green.

of collimated CT scan data using superDimension ${ }^{\mathrm{TM}}$ software (Covidien, Minneapolis, MN, USA) to create a virtual bronchoscopic pathway to the target lesion. In the operating room, sensors are attached to the patient which are then registered with an electromagnetic bronchoscopy system. Virtual-guided bronchoscopy is then performed to within a goal distance of $1 \mathrm{~cm}$ or less to the target lesion and ICG contrast is injected via a bronchoscopic needle. At our institution, $0.5 \mathrm{~mL}$ of ICG solution is injected followed by a flush of $0.5 \mathrm{~mL}$ of sterile water. During robotic surgery on the $\mathrm{Xi}$ version of the da Vinci robotic system, the thoracoscopic camera is equipped with near-infrared technology (Firefly, Intuitive Surgical, Sunnyvale, CA, USA) and can identify the lesion (Figure 2).

In 2001, Sakamoto et al. were the first to describe pulmonary nodule localization using indigo carmine contrast injected via flexible bronchoscopy (25). A series of studies followed, reporting accurate localization of small pulmonary nodules using electromagnetic navigation bronchoscopy (ENB) with transbronchial injection of blue dyes, most commonly, methylene blue (26). While the accuracy of blue dye contrasts range from $79-100 \%$, these agents are limited by quick diffusion in lung tissue, thereby limiting precision intraoperative visualization. A number of groups have conducted institutional studies regarding the accuracy of ENB-guided NIF using ICG for intraoperative localization. In 2014, Okusanya et al. conducted a two-stage trial with preoperative intravenous ICG $(5 \mathrm{mg} / \mathrm{kg}$ ) followed by open thoracotomy within 24 hours (27). NIF detected 16/18
(88.9\%) nodules. Furthermore, five additional nodules were identified which were not initially apparent on CT imaging. The authors found that the sensitivity for detecting nodules with ICG was dependent on tissue depth but independent of nodule size, metabolic activity, histology, or vascularity. In 2018, Anayama and colleagues reported a series of 37 patients with small pulmonary nodules who underwent thoracoscopic wedge resection using a mixture of ICG and iopamidol for localization by either CT-guided percutaneous injection or bronchoscopic injection (28). In the CT-guided group, 15/15 $(100 \%)$ nodules were successfully and in the bronchoscopic group, 20/22 (90.9\%) nodules were localized accurately.

Our institutional experience with NIF localization using ICG when administered both via ENB and intravenous injection has been previously detailed (29). In a cohort of patients undergoing robotic segmentectomy, 93 were selected for ENB localization with ICG. Lesions were localized based on size (favored for small lesions $<2 \mathrm{~cm}$ ), morphology (ground-glass opacification), and anatomy (favored in lesions deep to the pleural surface). Of these 93 patients, the pulmonary nodule was successfully identified in 80 patients $(86 \%)$. The most common reasons for localization failure were inaccurate electromagnetic navigational bronchoscopy or ICG injection (due to inaccurate mapping) and technical malfunctions of the equipment (inability to calibrate the electromagnetic field or inability to load the mapping data). Key factors to decrease inaccurate ICG injection include proper patient selection, as nodules far from an airway will be difficult to localize, and injection on the proximal aspect of peripheral nodules, as distal injection can lead to ICG infiltrating the pleural space.

\section{Image guided video-assisted thoracic surgery (iVATS)}

iVATS involves placement of a T-bar in small pulmonary nodules at the time of the operation in a hybrid operating room. This specialized room includes both a C-arm fluoroscopic imaging system as well as full equipment for an operating room. This intraoperative procedure eliminates patient transport between two locations. In the first clinical trial, there was $100 \%$ tagging on nodules with complete resection, with an average lesion size of $13 \pm 3.8 \mathrm{~mm}$ (30). A subsequent larger study of 50 patients from the same group, Gill et al., demonstrated a continued high success rate of 97\% utilizing this technique, with six different surgeons included in the study (31). 
Table 1 Comparison of localization techniques for small pulmonary nodules

\begin{tabular}{|c|c|c|c|}
\hline Localization technique & Advantages & Disadvantages & Equipment needed \\
\hline \multicolumn{4}{|l|}{ Preoperative markers } \\
\hline Microcoil & $\begin{array}{l}\text { No protruding wire } \\
\text { Can be performed a separate day }\end{array}$ & Requires fluoroscopic identification & $\begin{array}{l}\text { CT guidance by IR } \\
\text { Intraoperative fluoroscopy }\end{array}$ \\
\hline Hookwire & Easy to visualize the wire going to the nodule & $\begin{array}{l}\text { Must be performed the same day of OR } \\
\text { Risk of air embolism }\end{array}$ & CT guidance by IR \\
\hline Fiducial & $\begin{array}{l}\text { No protruding wire } \\
\text { Can be performed a separate day } \\
\text { Easier to place than microcoil }\end{array}$ & $\begin{array}{l}\text { Requires fluoroscopic identification } \\
\text { Risk of vascular embolization }\end{array}$ & $\begin{array}{l}\text { CT guidance by IR } \\
\text { Intraoperative fluoroscopy }\end{array}$ \\
\hline \multicolumn{4}{|l|}{ Preoperative liquids } \\
\hline Methylene blue & $\begin{array}{l}\text { Visualize easily without special } \\
\text { equipment }\end{array}$ & $\begin{array}{l}\text { Rapid diffusion into tissue, so must be on } \\
\text { same day as OR } \\
\text { Anaphylactic reaction }\end{array}$ & CT guidance by IR \\
\hline Tc-99m & No need for fluoroscopy & $\begin{array}{l}\text { Rapid diffusion into tissue, so must be on } \\
\text { same day as OR } \\
\text { Radiation exposure }\end{array}$ & $\begin{array}{l}\text { CT guidance by IR } \\
\text { Radiotracer } \\
\text { Gamma probe }\end{array}$ \\
\hline \multicolumn{4}{|l|}{ Intraoperative techniques } \\
\hline NIF with ICG & $\begin{array}{l}\text { Performed by surgeon } \\
\text { Same OR for tagging and surgery }\end{array}$ & $\begin{array}{l}\text { Requires surgeon expertise at navigational } \\
\text { bronchoscopy }\end{array}$ & $\begin{array}{l}\text { Navigational bronchoscopy } \\
\text { NIF camera }\end{array}$ \\
\hline \multirow[t]{2}{*}{ iVATS } & Same OR for tagging and surgery & $\begin{array}{l}\text { Requires radiologist availability at } \\
\text { time of procedure }\end{array}$ & Hybrid room \\
\hline & $\begin{array}{l}\text { Easy to visualize the wire going to the nodule } \\
\text { Patient in the same position for tagging and } \\
\text { resection }\end{array}$ & Dislodged T-bars & $\begin{array}{l}\text { CT guidance software and } \\
\text { radiologist }\end{array}$ \\
\hline
\end{tabular}

CT, computed tomography; IR, interventional radiology; OR, operating room; Tc-99m, technetium-99m; NIF, near-infrared fluorescence; ICG, indocyanine green contrast; iVATS, image guided video-assisted thoracic surgery.

Since iVATS is a relatively new technique, mostly described at a single center, the main risks associated are still being discovered. The combined studies demonstrated a low rate of postoperative pneumonia, prolonged air leak, one patient with an ileus and one patient with a splenic bleed requiring embolization $(30,31)$. Due to the immediate transition from $\mathrm{T}$ bar placement to surgical resection, the risk of clinically significant pneumothoraxes was not noted $(30,31)$. However, as with other markers, the $\mathrm{T}$ bar can be dislodged. In the larger series of patients, $3 \mathrm{~T}$ bars were displaced into the diaphragm, chest wall, or liver (31). However, due to entry marks on the visceral pleura, the nodules were still identified and resected during the VATS.

\section{Conclusions}

The number of small pulmonary nodules that require surgical resection for diagnosis will only continue to increase due to the widespread use of CT scans. With pulmonary resections being done via minimally invasive techniques, thoracic surgeons must be familiar with the different techniques of localizing these lesions for successful resection (Table 1). While most techniques utilize preoperative image guided placement of markers or injection of liquids, newer technology is making concurrent localization and resection the direction of the future. However, these newer technologies need either surgeon expertise in navigational bronchoscopy or available hybrid operating rooms. If either of those are not available, preoperative localization requires the support of the interventional radiology department. The exact modality used to help identify these lesions will depend on the experience and resources available at each center. However, each of these techniques is an overall reliable and safe way to aid in surgical resection of small pulmonary nodules. 


\section{Acknowledgments}

Funding: None.

\section{Footnote}

Provenance and Peer Review: This article was commissioned by the Guest Editors (Scott Swanson, Daniel Dolan) for the series "How to Evaluate, Diagnose and Treat Small Lung Nodules" published in Current Challenges in Thoracic Surgery. The article has undergone external peer review.

Reporting Checklist: The authors have completed the Narrative Review reporting checklist. Available at https:// ccts.amegroups.com/article/view/10.21037/ccts-20-90/rc

Peer Review File: Available at https://ccts.amegroups.com/ article/view/10.21037/ccts-20-90/prf

Conflicts of Interest: All authors have completed the ICMJE uniform disclosure form (available at https://ccts. amegroups.com/article/view/10.21037/ccts-20-90/coif). The series "How to Evaluate, Diagnose and Treat Small Lung Nodules" was commissioned by the editorial office without any funding or sponsorship. RJC reports personal fees from Intuitive, outside the submitted work. The authors have no other conflicts of interest to declare.

Ethical Statement: The authors are accountable for all aspects of the work in ensuring that questions related to the accuracy or integrity of any part of the work are appropriately investigated and resolved.

Open Access Statement: This is an Open Access article distributed in accordance with the Creative Commons Attribution-NonCommercial-NoDerivs 4.0 International License (CC BY-NC-ND 4.0), which permits the noncommercial replication and distribution of the article with the strict proviso that no changes or edits are made and the original work is properly cited (including links to both the formal publication through the relevant DOI and the license). See: https://creativecommons.org/ licenses/by-nc-nd/4.0/.

\section{References}

1. National Lung Screening Trial Research Team, Aberle DR, Adams AM, et al. Reduced lung-cancer mortality with low-dose computed tomographic screening. N Engl J Med 2011;365:395-409.

2. Kang MC, Kang CH, Lee JH, et al. Accuracy of 16-channel multi-detector row-chest computed tomography with thin sections in the detection of metastatic pulmonary nodules. Eur J Cardiothorac Surg 2008;33:473-9.

3. Blanke P, Schoepf UJ, Leipsic JA. CT in transcatheter aortic valve replacement. Radiology 2013;269:650-69.

4. Lehman SJ, Abbara S, Cury RC, et al. Significant of cardiac computed tomography incidental findings in acute chest pain. Am J Med 2009;122:543-9.

5. Suzuki K, Nagai K, Yoshida J, et al. Video-assisted thoracoscopic surgery for small indeterminate pulmonary nodules: indications for preoperative marking. Chest 1999;115:563-8.

6. McDermott S, Fintelmann FJ, Bierhals AJ, et al. Imageguided preoperative localization of pulmonary nodules for video-assisted and robotically assisted surgery. Radiographics 2019;39:1264-79.

7. Donahoe LL, Nguyen ET, Chung TB, et al. CT-guided microcoil VATS resection of lung nodules: a single-centre experience and review of the literature. J Thorac Dis 2016;8:1986-94.

8. Rodrigues JCL, Pierre AF, Hanneman K, et al. CT-guided microcoil pulmonary nodule localization prior to videoassisted thoracoscopic surgery: diagnostic utility and recurrence-free survival. Radiology 2019;291:214-22.

9. Finley RJ, Mayo JR, Grant K, et al. Preoperative computed tomography-guided microcoil localization of small peripheral pulmonary nodules: a prospective randomized controlled trial. J Thorac Cardiovasc Surg 2015;149:26-31.

10. Powell TI, Jangra D, Clifton JC, et al. Peripheral lung nodules: fluoroscopically guided video-assisted thoracoscopic resection after computed tomographyguided localization using platinum microcoils. Ann Surg 2004;240:481-8.

11. Zhong Y, Xu XQ, Pan X L, et al. Retrospective evaluation of safety, efficacy and risk factors for pneumothorax in simultaneous localizations of multiple pulmonary nodules using hook wire system. Cardiovasc Intervent Radiol 2017;40:1408-14.

12. Ciriaco P, Negri G, Puglisi A, et al. Video-assisted thoracoscopic surgery for pulmonary nodules: rationale for preoperative computed tomography-guided hookwire localization. Eur J Cardiothorac Surg 2004;25:429-33.

13. Ichinose J, Kohno T, Fuijmori S, et al. Efficacy and complication of computer tomography-guided hook wire 
localization. Ann Thorac Surg 2013;96:1203-8.

14. Seo JM, Lee HY, Kim HK, et al. Factors determining successful computer tomography-guided localization of lung nodules. J Thorac Cardiovasc Surg 2012;143:809-14.

15. Kleedehn M, Kim DH, Lee FT, et al. Preoperative pulmonary nodule localization: a comparison of methylene blue and hookwire techniques. AJR Am J Roentgenol 2016;207:1334-9.

16. Horan TA, Pinheiro PM, Araujo LM, et al. Massive gas embolism during pulmonary nodule hook wire localization. Ann Thorac Surg 2002;73:1647-9.

17. Sancheti MS, Lee R, Ahmed SU, et al. Percutaneous fiducial localization for thoracoscopic wedge resection of small pulmonary nodules. Ann Thorac Surg 2014;97:1914-8.

18. Sharma A, McDermott S, Mathisen DJ, et al. Preoperative localization of lung nodules with fiducial markers: feasibility and technical considerations. Ann Thorac Surg 2017;103:1114-20.

19. Cousin F, Gérard L, Joskin J. Left cardiac migration of a lung fiducial marker. J Vasc Interv Radiol 2019;30:445.

20. Vandoni RE, Cuttat JF, Wicky S, et al. CT-guided methyleneblue labelling before thoracoscopic resection of pulmonary nodules. Eur J Cardiothorac Surg 1998;14:265-70.

21. Shentu Y, Zhang L, Gu H, et al. A new technique combining virtual simulation and methylene blue staining for the localization of small peripheral pulmonary lesions. BMC Cancer 2014;14:79.

22. Ramin S, Azar FP, Malihe H. Methylene blue as the safest blue dye for sentinel node mapping: emphasis on anaphylaxis reaction. Acta Oncologica 2011;50:729-31.

23. Daniel, TM, Altes TA, Rehm PK, et al. A Novel

doi: $10.21037 /$ ccts-20-90

Cite this article as: Chang SH, Geraci TC, Johnson KR, Kent AJ, Cerfolio RJ. Narrative review: preoperative localization techniques for small lung nodules. Curr Chall Thorac Surg 2022;4:36.
Technique for Localization and Excisional Biopsy of Small or Ill-Defined Pulmonary Lesions. Ann Thorac Surg 2004;77:1756-62.

24. Ambrogi MC, Melfi F, Zirafa C, et al. Radio-guided thoracoscopic surgery (RGTS) of small pulmonary nodules. Surg Endosc 2012;26:914-9.

25. Sakamoto T, Takada Y, Endoh M, et al. Bronchoscopic dye injection for localization of small pulmonary nodules in thoracoscopic surgery. Ann Thorac Surg 2001;72:296-7.

26. Cuadrado DG, Grogan EL. Localization techniques for small lung nodules. Oper Tech Thorac Cardiovasc Surg 2014;19:179-98.

27. Okusanya OT, Holt D, Heitjan D, et al. Intraoperative near-infrared imaging can identify pulmonary nodules. Ann Thorac Surg 2014;98:1223-30.

28. Anayama T, Hirohashi K, Miyazaki R, et al. Near-infrared dye marking for thoracoscopic resection of small-sized pulmonary nodules: comparison of percutaneous and bronchoscopic injection techniques. J Cardiothorac Surg 2018;13:5.

29. Geraci TC, Ferrari-Light D, Kent A, et al. Technique, outcomes with navigational bronchoscopy using indocyanine green for robotic segmentectomy. Ann Thorac Surg 2019;108:363-9.

30. Gill RR, Zheng Y, Barlow JS, et al. Image-guided video assisted thoracoscopic surgery (iVATS) - phase I-II clinical trial. J Surg Oncol 2015;112:18-25.

31. Gill RR, Barlow J, Jaklitsch MT, et al. Image-guided videoassisted thoracoscopic resection (iVATS): Translation to clinical practice- real-world experience. J Surg Oncol 2020;121:1225-32. 